\title{
SCANNING TUNNELING MICROSCOPY OF ORDERED ORGANIC MONOLAYER FILMS ON Si(001)
}

\author{
R.J. Hamers, J. Hovis and H. LiU \\ Department of Chemistry, University of Wisconsin \\ 1101 University Avenue, Madison, WI 53706, USA
}

Scanning tunneling microscopy and optical spectroscopy techniques have been utilized to investigate the formation of ordered organic monolayer films on the (001) face of silicon. Cyclopentene and 1,5-cyclooctadiene both produce monolayer films that are ordered translationally and rotationally. The rotational orientations of the molecules arise from the directional interaction of the $\pi$ orbitals of the starting alkene with the $\pi$ orbital of the dimers comprising the reconstructed $\mathrm{Si}(001)$ surface, with the $\mathrm{Si}(001)$ surface acting as a template for determining the directionality of molecules in the subsequent organic film. Using single-domain $\mathrm{Si}(001)$ samples, it is shown that the molecular films also exhibit anisotropy in optical properties when measured on centimeter length scales.

PACS numbers: $61.16 . \mathrm{Ch}, 34.50 . \mathrm{Dy}, 68.55 .-\mathrm{a}$

\section{Introduction}

It is now widely recognized that scanning tunneling microscopy (STM) images reflect the local electronic structure of the surface under investigation. On consequence of this is that it becomes difficult to establish a definitive relationship between the structure of a molecule and its appearance in the STM. From the standpoint of electronic structure, one of the simplest and most well-studied surfaces is the (001) surface of silicon, widely utilized in the microelectronics industry. The Si(001) surface is characterized by pairs of atoms bonded together to form dimers [1]. Formally, at least, the silicon atoms within each dimer are held together with both a strong sigma bond and a much weaker $\pi$ bond. In coordination chemistry, it is generally recognized that the orbitals which control chemical reactivity are the highest-occupied molecular orbital (HOMO) and the lowest unoccupied molecular orbital (LUMO). Scanning tunneling microscope images of the filled and empty states of the Si(001) surface show local structures that confirm this molecular orbital picture. 


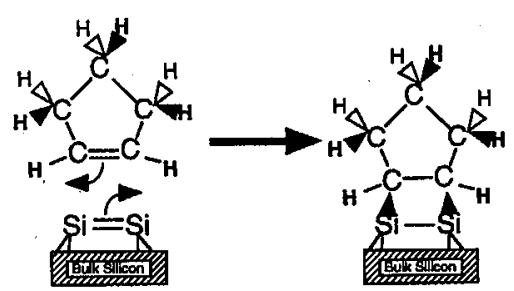

Fig. 1. Schematic depiction of cycloaddition reaction between $\mathrm{Si}=\mathrm{Si}$ dimers or $\mathrm{Si}(001)$ surface and an unsaturated alkene, cyclopentene.

Formation of ordered organic films on $\mathrm{Si}(001)$ surface typically involves fragmentation of the organic molecule. In the specific case of unsaturated organic molecules however, another mode of bonding is possible. As depicted in Fig. 1, alkenes (molecules having one or more double bonds) can bond to the $\operatorname{Si}(001)$ surface by breaking the $\pi$ bonds of the alkene and the $\mathrm{Si}(001)$ dimers and forming two new sigma $(\sigma)$ bonds. This mode of bonding is interesting and unique because it does not involve fragmentation of the organic molecule and therefore provides better control of the atomic-level structure than dissociative reactions would. Previous studies have shown that this mode of bonding is utilized in the bonding of ethylene and acetylene to the $\mathrm{Si}(001)$ surface [2-8]. In recent studies, we have investigated the adsorption and atomic-level structures formed by the interaction of unsaturated organic molecules with the $\operatorname{Si}(001)$ surface $[9,10]$.

\section{Experimental}

Experiments were performed in an ultrahigh vacuum chamber with a nominal pressure of $10^{-10} \operatorname{Tr}$. Si $(001)$ samples were cleaned by high-temperature annealing to $1500 \mathrm{~K}$, followed by cooling to room temperature. The scanning tunneling microscope is a home-built design. Infrared spectra was obtained in an ultrahigh vacuum chamber equipped with $\mathrm{BaF}_{2}$ windows, using a commercial FTIR spectrometer and an $\mathrm{InSb}$ detector. Cyclopentene and 1,5-cyclooctadiene were high-purity forms (Aldrich Chemical) and used as received.

\section{Results and discussion}

Figure 2 shows an STM image of the Si(001) surface after exposure to saturation coverage of cyclopentene (already depicted schematically in Fig. 1). In the image, it can be seen that the molecules are aligned into rows just like the underlying Si(001) surface. In fact, the inherent directionality of the $\pi$ orbitals of both $\mathrm{Si}=\mathrm{Si}$ dimers and the alkene results in the organic molecules not only be adsorbed into an ordered array, but also having their rotational orientation controlled by that of the underlying surface. Thus, the surface $\mathrm{Si}=\mathrm{Si}$ dimers essentially act as "templates" for controlling the translational and rotational ordering of the organic monolayer.

One interesting property of the $\mathrm{Si}(001)$ surface is that it is possible to some extent to control the orientation of the surface dimers. On samples cut closely along the (001) plane (as in Fig. 2), the steps are far apart and the crystallography 
of the $\mathrm{Si}$ lattice structure dictates that the $\mathrm{Si}=\mathrm{Si}$ dimers (and consequently the organic molecules) must rotate in orientation across each step. However, purposely miscutting the $\mathrm{Si}(001)$ by approximately $4^{\circ}$ leads to a preponderance of steps that are two atoms high [11]; across such "bilayer" steps the dimers retain their orientation, making it possible to prepare samples in which the organic molecules are rotationally aligned on macroscopic dimensions. We have recently shown that this in turn leads to anisotropy in optical properties [10,1]. Films of this nature might also exhibit interesting anisotropy in other properties such as electrical conductivity, although we have not yet explored these properties.

In Figs. 1-3, the molecules appear elongated. This elongation is interpreted as reflecting the direction of the nearly planar ring structure. To explore the relationship between the STM images and the molecular structure, it is useful to compare these images with those of a related molecule, 1,5-cyclooctadiene (COD).

Figure 4 shows a schematic illustration of the 1,5-cyclooctadiene molecule. In that gas phase, this molecule adopts a "twist-boat" configuration in which the two $\mathrm{C}=\mathrm{C}$ groups are slightly twisted with respect to one another. Figure 5 shows STM images of $\mathrm{Si}(001)$ after exposure to COD. Upon first inspection of the molecule structure it might be thought that this molecule has the potential for bonding to the $\operatorname{Si}(001)$ surface using both $\pi$ bonds; however, bonding via both $\pi$ systems results in poor overlap of the $\pi$ molecular orbitals of the molecule with those of the $\mathrm{Si}=\mathrm{Si}$ dimers. In Fig. 5 , it can be seen that the molecules are adsorbed into a highly ordered array. Unlike the previous case of cyclopentene, however, the COD molecules show a significant amount of internal structure that can be observed more clearly in the high resolution image at the bottom part of Fig. 5.

Further insight into the relationship between the molecular structure and the STM images requires further chemical information about the molecules on the surface. The differences in STM images of cyclopentene and cyclooctadiene suggest that they may be some intrinsic differences in the exposed chemical functional

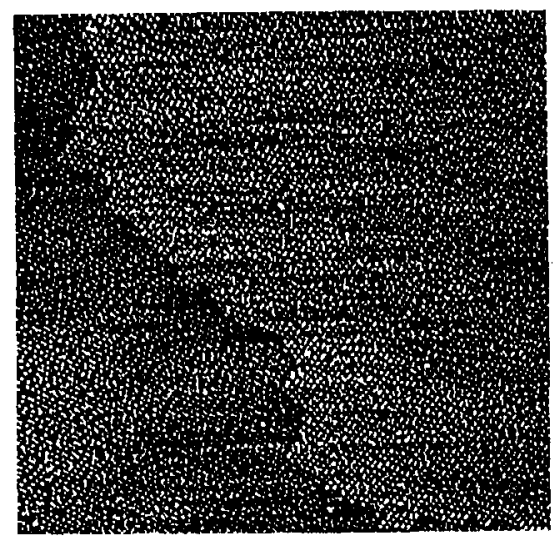

Fig. 2. STM images of cyclopentene on Si(001), including a single-height atomic step. 

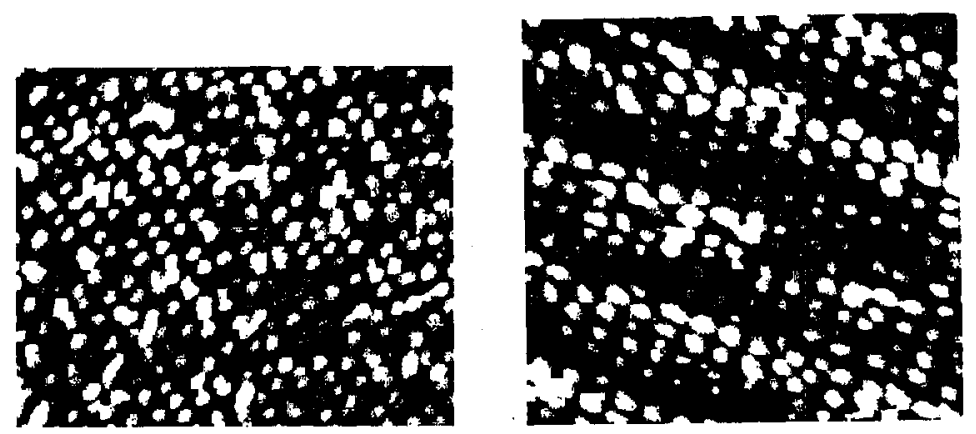

Fig. 3. STM images of cyclopentene adsorbed on flat (left) and $4^{\circ}$-miscut vicinal $\mathrm{Si}(001)$ (right). In both cases, the molecules appear as elongated bean-shaped protrusions with no other internal structure.

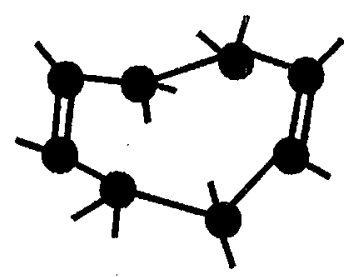

Fig. 4. Schematic illustration of 1,5-cyclooctadiene. Unterminated lines represent C-H bonds.

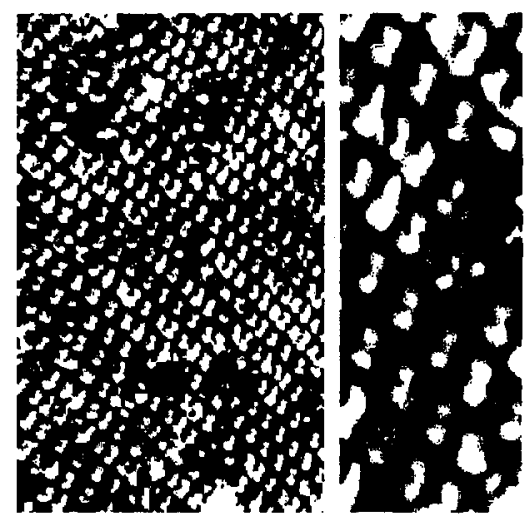

Fig. 5. STM images of cyclooctadiene adsorbed on Si(001) at low resolution (left) and at high resolution (right). The STM images show that the molecules are highly ordered and that each molecule gives rise to two protrusions.

groups. To prove this requires direct chemical information. Because the separation between $\pi$ bonding and $\pi^{*}$ antibonding levels is smaller than the separation between $\sigma$ bonding and $\sigma^{*}$ antibonding levels, one would expect to see systematic differences in tunneling current-voltage characteristic measured on alkane-like 
regions and on alkene-like regions. Unfortunately, we found that the stability of STM images on these organic films was also very dependent on the applied voltage. Good-quality, stable images were only obtained at negative sample voltage (filled state images) and at low currents $(<200 \mathrm{pA})$. Even momentary exposure to higher voltages or currents has the effect of destabilizing the microscope, most likely by inducing further chemical transformations in the organic films.

While conventional tunneling spectroscopy methods are not easily applied to these organic films, an alternative method for obtaining chemical information at an atomic level is to integrate spatial and chemical probes in a way that permits results of the two techniques to be correlated with one another. When single-domain samples are utilized, then FTIR measurements along different polarization directions can be used to provide detailed information about the geometric structure of the molecule. To this end, we have integrated Fourier-transform infrared spectroscopy (FTIR) with STM. Figure 6 shows FTIR spectra measured with the electric field vector of the infrared radiation parallel to the surface plane. In Fig. 6, direction " $\mathrm{A}$ " corresponds to having the electric field parallel to the surface plane but perpendicular to the $\mathrm{Si}=\mathrm{Si}$ bond axis, while direction " $\mathrm{B}$ " corresponds to having the electric field vector parallel to the surface plane and parallel to the $\mathrm{Si}=\mathrm{Si}$ dimer bond axis. While the STM images in Fig. 5 clearly show that the molecules are highly oriented on a microscopic length scale, the differences between the spectra " $\mathrm{A}$ " and " $\mathrm{B}$ " demonstrate that the molecules are oriented on length scales of centimeters. The features at around $2850-2980 \mathrm{~cm}^{-1}$ arise from alkane-like $\mathrm{C}-\mathrm{H}$ stretching vibrations while the very sharp feature near $3020 \mathrm{~cm}^{-1}$ is characteristic of the $\mathrm{C}-\mathrm{H}$ stretching mode of an alkene (i.e., a $\mathrm{C}=\mathrm{C}-\mathrm{H}$ vibration). The presence of this relatively large peak at $3020 \mathrm{~cm}^{-1}$ demonstrates that there are alkene-like groups present even after the COD molecule is bonded on the Si surface. Finally, we note that there is almost no absorption present in the $2060-2080 \mathrm{~cm}^{-1}$ region, which is the region where $\mathrm{Si}-\mathrm{H}$ stretching vibrations are commonly observed. The absence of any significant absorbance in this region demonstrates that the COD molecules adsorb on the surface without breaking any $\mathrm{C}-\mathrm{H}$ bonds.

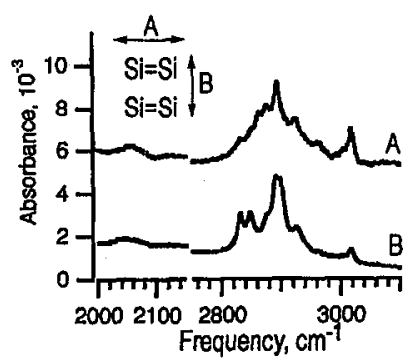

Fig. 6. Fourier-transform infrared spectra of $\mathrm{Si}(001)$ after exposure to 1,5-cyclooctadiene. The electric field vector of the light is in the plane of the surface and is perpendicular to the dimer bond axis " $\mathrm{A}$ " and parallel to the dimer bond axis " $\mathrm{B}$ ". 


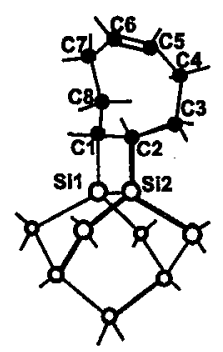

Fig. 7. Bonding geometry of 1,5-cyclooctadiene on a 9-atom Si cluster representing the $\mathrm{Si}(001)$ surface. Black circles represent carbon atoms, open circles represent Si atoms. Note that only one $\mathrm{C}=\mathrm{C}$ bond is used in bonding to the substrate, leaving the second unsaturated $\mathrm{C}=\mathrm{C}$ bond exposed at the interface with the vacuum.

The infrared spectra clearly show that only one end of the COD molecule binds directly to the surface, with the second $\mathrm{C}=\mathrm{C}$ bond exposed at the vacuum, as depicted in Fig. 7. This particular geometry is interesting because it implies that the surfaces shown in Fig. 5 are ordered arrays of $\mathrm{C}=\mathrm{C}$ double bonds. There exists a rich variety of reactions in the organic chemistry dealing with alkene groups, suggesting that surfaces like these might be readily functionalized to other groups using standard organic chemistry reactions.

The differences between STM images of cyclopentene and cyclooctadiene suggest that STM may be able to distinguish between alkane groups and alkene groups, due to the fact that STM senses local electronic, not structural, properties. At the present time it is not understood why each COD molecule gives rise to two protrusions when the filled states are imaged. The electronic state density of the $\mathrm{C}=\mathrm{C} \pi$ bond is expected to reach a single maximum at the center of $\mathrm{C}=\mathrm{C}$ bond. The observation of two protrusions then suggests that perhaps the $\mathrm{C}-\mathrm{H}$ electronic states are playing an important role in determining the STM images. Continued efforts are underway to understand this utilizing electronic structure calculations.

\section{Conclusions}

The application of STM to investigate organic molecules is yet in its infancy. In any chemical system, however, it is imperative that both the "physical" and "chemical" structures be identified. Here, we have shown that STM is able to distinguish between different organic molecules having different chemical structures on $\mathrm{Si}(001)$. It appears that unsaturated bonds (as in cyclooctadiene) have a different appearance than saturated bonds (as in cyclopentene after adsorption on $\mathrm{Si}(001)$ ). In more recent studies not included here, we have found the unsaturated molecule cyclooctatetraene, also orders and shows a bifurcation along the unsaturated $\mathrm{C}=\mathrm{C}$ bond similar to that of 1,5-cyclooctadiene.

This work was supported by the National Science Foundation and by the U.S. Office of Naval Research. 


\section{References}

[1] R.J. Hamers, R.M. Tromp, J.E. Demuth, Phys. Rev. B 34, 5343 (1986).

[2] W. Widdra, C. Huang, W.H. Weinberg, Surf. Sci. 329, 295 (1995).

[3] W. Widdra, C. Huang, S.I. Yi, W.H. Weinberg, J. Chem. Phys. 105, 5605 (1996).

[4] A.J. Mayne, T.R.I. Cataldi, J. Knall, A.R. Avery, T.S. Jones, L. Pinheiro, H.A.O. Hill, G.A.D. Briggs, J.B. Pethica, W.H. Weinberg, Faraday Discuss. $R$. Soc. 94, 199 (1992).

[5] A.J. Mayne, A.R. Avery, J. Knall, T.S. Jones, G.A.D. Briggs, W.H. Weinberg, Surf. Sci. 284, 247 (1993).

[6] L. Clemen, R.M. Wallace, P.A. Taylor, M.J. Dresser, W.J. Choyke, W.H. Weinberg, J.T.Jr. Yates, Surf. Sci. 268, 205 (1992).

[7] C.C. Cheng, R.M. Wallace, P.A. Taylor, W.J. Choyke, J.T.Jr. Yates, J. Appl. Phys. 67, 3693 (1990).

[8] A.J. Fisher, P.E. Blochl, G.A.D. Briggs, Surf. Sci. 374, 298 (1997).

[9] R.J. Hamers, J.S. Hovis, S. Lee, H. Liu, J. Shan, J. Phys. Chem. B 101, 1489 (1397).

[10] J.S. Hovis, S. Lee, H. Liu, R.J. Hamers, J. Vac. Sci. Technol. A, 1997, accepted for publication.

[11] J.D. Chadi, Phys. Rev. Lett. 59, 1691 (1987). 